\title{
LSD1 negatively regulates autophagy through the mTOR signaling pathway in ovarian cancer cells
}

\author{
YE WEI, TIANTIAN HAN, RANRAN WANG, JING WEI, KE PENG, QIONG LIN and GENBAO SHAO \\ School of Medicine, Jiangsu University, Zhenjiang, Jiangsu 212013, P.R. China
}

Received November 29, 2017; Accepted April 11, 2018

DOI: $10.3892 /$ or.2018.6432

\begin{abstract}
Lysine-specific demethylase 1 (LSD1) plays a key role in cell proliferation, differentiation and carcinogenesis. In the present study we revealed that LSD1 functioned as an autophagy suppressor in ovarian cancer HO8910 cells. Pharmacological inhibition or genetic knockdown of LSD1 resulted in the elevation of the LC3-II protein, enhancement of autophagosomal formation and stimulation of the autophagic flux. In addition, knockdown of LSD1 further promoted the serum starvation- and rapamycin-induced autophagy. Furthermore, we demonstrated that LSD1 regulated autophagy via the mTOR signaling pathway. Collectively, our findings identified LSD1 as a novel negative regulator of autophagy through the mTOR signaling pathway in ovarian cancer HO8910 cells and indicated that LSD1 may function as a driving factor of ovarian cancer progression via deregulating autophagy.
\end{abstract}

\section{Introduction}

Histone modification represents one of the main mechanisms of the epigenetic regulation of gene expression $(1,2)$. Increasing evidence indicated that specific members of the histone demethylase family have been associated with human diseases, especially carcinogenesis (3). Notably, LSD1 was the first discovered histone demethylase which specifically catalyzed the removal of mono- and dimethyl groups from methylated histone $\mathrm{H} 3$ at lysine $4(\mathrm{H} 3 \mathrm{~K} 4 \mathrm{mel} / 2)$ contributing to silencing or activation of target genes (4). LSD1 is highly expressed in a variety of carcinomas, including breast (5), lung (6), hepatocarcinoma (7) and ovarian cancer (8). Our previous studies have demonstrated that the overexpression of LSD1 was associated with an aggressive phenotype and poor prognosis of ovarian cancer (9). However, the molecular

Correspondence to: Dr Genbao Shao or Professor Qiong Lin, School of Medicine, Jiangsu University, 301 Xuefu Road, Zhenjiang, Jiangsu 212013, P.R. China

E-mail: gbshao07@ujs.edu.cn

E-mail: humandock11@gmail.com

Key words: LSD1, LC3-II, autophagy, mTOR signaling, ovarian cancer mechanism underlying the association of the overexpression of LSD1 with the progression of ovarian cancer remains largely unknown.

Autophagy is an evolutionarily conserved cellular process for recycling and removal of damaged organelles, macromolecules and protein aggregates which are encapsulated by double-membrane structures (autophagosomes) and delivered to lysosomes for degradation (10-12). Autophagy is essential for cells to gain energy and maintain cellular homeostasis, which protects them from nutrient and environmental stress (13). Microtubule-associated protein 1 light chain 3 (LC3) is a protein participating in autophagy through conjugation with phosphatidylethanolamine (PE) to form LC3-II, which is an autophagosomal marker $(14,15)$. Activation of autophagy is sensitized by inactivation of the mammalian target of rapamycin (mTOR), which is a major cellular nutrition and energy sensor kinase (16). Many studies have revealed that autophagy plays complex roles in tumorigenesis and tumor progression $(13,17,18)$. At present, how autophagy regulates tumorigenesis and tumor progression is not fully understood.

Numerous studies have revealed that autophagy was directly or indirectly regulated by epigenetics, either by the modification of transcriptional factors or chromatin structure during gene expression $(19,20)$. Recently, several studies found that LSD1 was involved in the regulation of autophagy through a variety of mechanisms $(21,22)$. In the present study, we observed that knockdown of LSD1 caused accumulation of LC3-II and markedly enhanced the autophagic flux in ovarian cancer HO8910 cells. Furthermore, depletion of LSD1 significantly promoted the starvation- and rapamycin-induced activation of autophagy. Notably, we found that serum starvation or rapamycin treatment downregulated LSD1, indicating that downregulation of LSD1 may mediate the activation of autophagy by starvation or rapamycin. Finally, we confirmed that the negative regulation of autophagy by LSD1 was conducted through the activation of the mTOR signaling pathway. Our study has provided direct evidence supporting the negative role of LSD1 in the regulation of autophagy in ovarian cancer cells.

\section{Materials and methods}

Cell lines and cell culture. The human ovarian epithelial cancer cells (HO8910) were kindly provided by Dr Qixiang Shao of Jiangsu University (Zhenjiang, China). HO8910 and 293 cells 
were cultured in Dulbecco's modified Eagle's medium (DMEM) containing $10 \%$ fetal bovine serum (FBS; both form Gibco, Grand Island, NY, USA) at $37^{\circ} \mathrm{C}$ in a humidified atmosphere containing $5 \% \mathrm{CO}_{2}$.

Reagents, antibodies and plasmids. pLKO-Tet-On,pHR'-CMV8.2 $\triangle \mathrm{VPR}$ and $\mathrm{pHR}$-CMV-VSVG vectors were kindly provided by Dr Changdeng Hu (Purdue University, West Lafayette, IN, USA). The antibodies were obtained as follows: the LSD1 (1:1,000; cat. no. 2184S), H3 (1:2,000; cat. no. 4499S), H3K4me2 (1:1,000; cat. no. 9725S), Beclin1 (1:1,000; cat. no. 3495P), LC3 (1:500; cat. no. 4180S), p70S6K (1:1,000; cat. no. 2708T) and phospho-p70S6K (1:1,000; cat. no. 9234T) antibodies were purchased from Cell Signaling Technology (Boston, MA, USA); $\alpha$-tubulin (1:5,000; cat. no. BS1699) and goat anti-rabbit IgG (H\&L)-HRP (1:5,000; cat. no. BS13278) were obtained from Bioworld Technology (Shanghai, China); Alexa Fluor ${ }^{\circledR} 488$ AffiniPure goat anti-rabbit IgG (H+L) (1:100; cat. no. R37116) were purchased from Invitrogen (Carlsbad, CA, USA). Electrochemiluminescence (ECL) reagents were purchased from Millipore (EMD Millipore, Billerica, MA, USA). Bioepitope Nuclear and Cytoplasmic Extraction kit were purchased from Bioworld Technology. Polybrene, doxycycline (Dox), chloroquine (CQ), tranylcypromine (TCP), dimethyl sulfoxide (DMSO), and dihydrochloride (DAPI) were purchased from Sigma-Aldrich (St. Louis, MO, USA). Bafilomycin A1 (BafA1) was purchased from GE Healthcare Life Sciences (Piscataway, NJ, USA). Rapamycin and MHY1485 were purchased from Selleck Chemicals (Shanghai, China).

Generation of stable LSD1 knockdown cell line. pLKOTet-On-shLSD1 plasmid and lentiviral particles were generated as previously described (9). Lipofectamine 2000 reagent (Invitrogen) was used as the transfection reagent and the transfection was performed according to the manufacturer's protocol. In brief, $1 \mathrm{ml}$ lentiviral supernatant and $3 \mathrm{ml}$ complete medium containing $8 \mu \mathrm{g} / \mathrm{ml}$ polybrene were added to infect the HO8910 cells. After being infected twice, $2 \mu \mathrm{g} / \mathrm{ml}$ puromycin was added into HO8910 cells for $72 \mathrm{~h}$ and then maintained with $1.0 \mu \mathrm{g} / \mathrm{ml}$ puromycin for two weeks to select stably transfected HO8910-shLSD1 cells.

$R N A$ extraction and $q R T-P C R$. RNA was extracted using the RNAiso plus (Takara Bio, Shiga, Japan). To produce cDNA, $2 \mu \mathrm{g}$ of total RNA was processed with the PrimeScript RT Reagent kit (Takara Bio) according to the manufacturer's instructions. Then the cDNAs were subjected to qRT-PCR as previously described (23). qRT-PCR was performed using the comparative cycle threshold (CT) method with SYBR-Green PCR Master Mix (Takara Bio) according to the manufacturer's instructions on a Bio-Rad CFX96 system (Bio-Rad Laboratories, Inc., Hercules, CA, USA). The primer sequences were used as follows: LSD1 (GenBank accession no. NM 015013.3), 5'-CAA GTGTCAATTTGTTCGGG-3' (forward) and 5'-TTCTTTGGG CTGAGGTACTG-3' (reverse), GAPDH (GenBank accession no. NM 001256799.1), 5'-GCAAATTCCATGGCACCGTC-3' (forward) and 5'-TCGCCCCACTTGATTTTGG-3' (reverse), LC3 (GenBank accession no. NM 022818.4), 5'-CCAGATCCC TGCACCATG-3' (forward) and 5'-CTGCTTCTCACCCTTGT ATCG-3' (reverse). The following PCR conditions were used: initial denaturation at $95^{\circ} \mathrm{C}$ for $30 \mathrm{sec}$, followed by 40 cycles of denaturation at $95^{\circ} \mathrm{C}$ for $5 \mathrm{sec}$, annealing at $58^{\circ} \mathrm{C}$ for $30 \mathrm{sec}$ and elongation at $72^{\circ} \mathrm{C}$ for $1 \mathrm{~min}$. The relative results were analyzed using the comparative cycle threshold method $\left(2^{-\Delta \Delta C T}\right)$ with $G A P D H$ as the reference gene (24).

Western blot analysis. Histones were prepared using the Bioepitope Nuclear and Cytoplasmic Extraction kit (Bioworld Technology) following the manufacturer's protocol. Cells were lysed with RIPA buffer (Kangchen Biotech, Shanghai, China) to extract total protein and equal amounts of soluble protein samples were subjected to 8-12\% SDS-PAGE gel and transferred to $0.2 / 0.45-\mu \mathrm{m}$ pore polyvinylidene difluoride (PVDF) membranes (EMD Millipore). The membranes were blocked in 5\% non-fat dry milk for $1 \mathrm{~h}$ at room temperature (RT). The membranes were washed in TBST three times and incubated overnight at $4^{\circ} \mathrm{C}$ with the corresponding primary antibodies. The membranes were then washed with TBST and incubated with the secondary antibody for $1 \mathrm{~h}$ at RT. The results were developed by ECL reagents. To perform densitometry analysis, digital images of the positive bands were obtained with ChemiDoc XRS and analyzed using the image analysis program Quantity One (BioRad Laboratories). The results were expressed as the ratio of target protein/loading control. Data shown are representative of three independent experiments.

Immunofluorescence. Cells were plated on glass coverslips in 24-well tissue culture plates. In brief, cells were washed with PBS, fixed with $4 \%$ paraformaldehyde and permeabilized with ice-cold 100\% methanol (25), and then the cells were blocked with 3\% bovine serum albumin (BSA) for $1 \mathrm{~h}$ at RT. The cells were incubated with primary antibody LC3 (1:200) at $4^{\circ} \mathrm{C}$ overnight and Alexa Fluor ${ }^{\circledR} 488$ AffiniPure goat anti-rabbit IgG $(\mathrm{H}+\mathrm{L})$ (1:100) was used as secondary antibody for $1 \mathrm{~h}$ at RT. The number of nuclei was revealed by DAPI staining. Fluorescence images were captured using the Nikon new Ni Series microscopes (Nikon, Melville, NY, USA).

Statistical analysis. All the experiments were repeated independently three times and the data values were presented as the mean \pm SEM. The differences between the groups were analyzed by Student's t-test, when two groups were compared or by one-way ANOVA, when more than two groups were compared using SPSS 11.5 software (SPSS, Inc., Chicago, IL, USA). P-values with a $95 \%$ confidence interval were obtained from at least three independent experiments. A P-value of $<0.05$ was considered to indicate a statistically significant difference.

\section{Results}

Knockdown of LSDI induces accumulation of LC3-II proteins. To examine whether LSD1 plays an important role in autophagy, we determined the effect of LSD1 knockdown on the level of the autophagosomal marker LC3-II. The inducible LSD1 shRNA cell line HO8910-shLSD1 was generated using lentiviral vector-loaded LSD1 shRNA in HO8910 ovarian cancer cells. As displayed in Fig. 1A and B, the protein product and mRNA levels of LSD1 were effectively silenced in HO8910-shLSD1 cells. In addition, LSD1 knockdown markedly increased the H3K4me2 levels, a major substrate of LSD1 


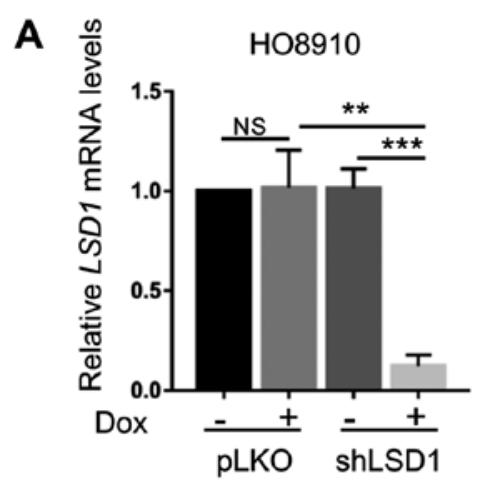

C

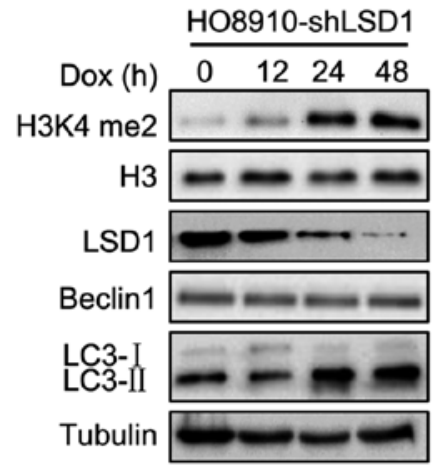

D

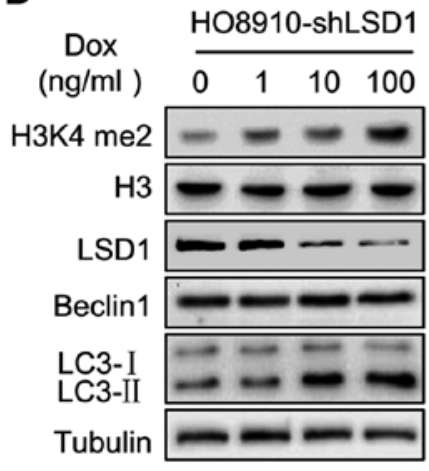

E

E

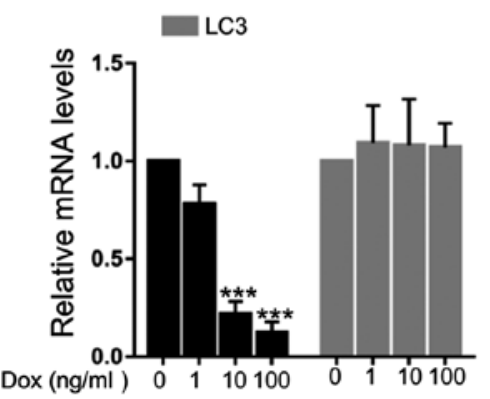

B
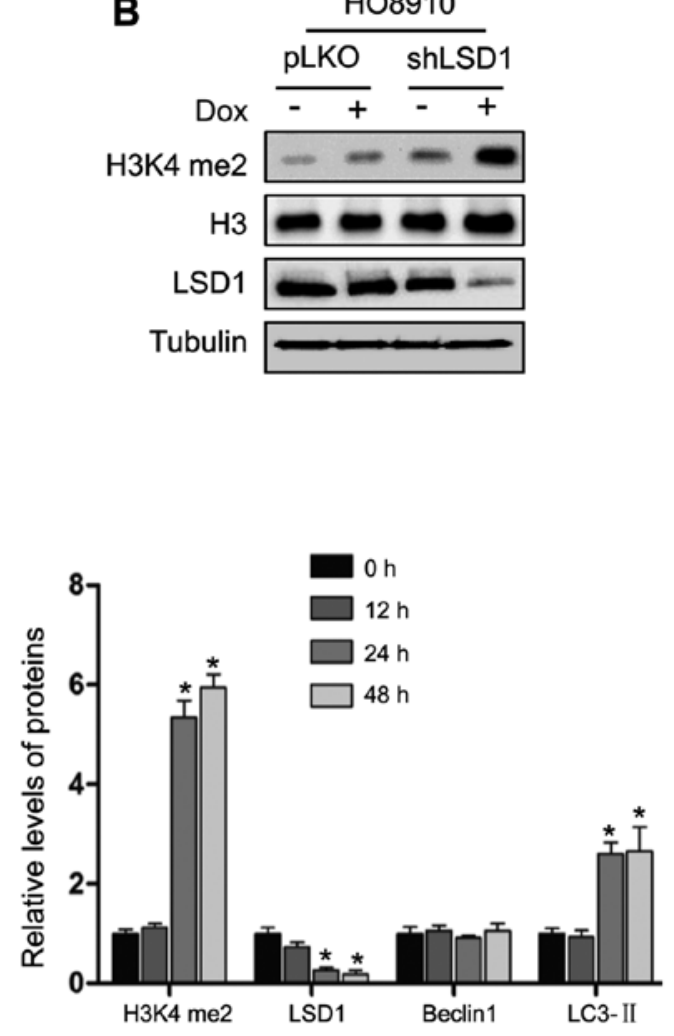

$\mathbf{F}$

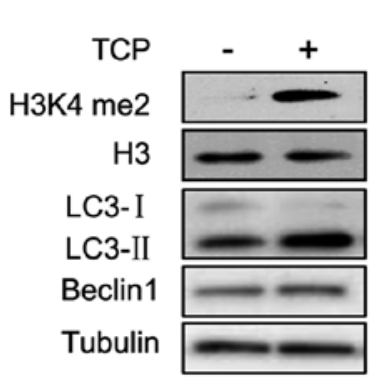

$\mathbf{G}$

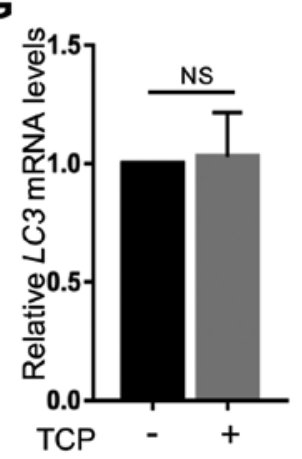

Figure 1. Knockdown of LSD1 induces accumulation of LC3-II proteins. (A) HO8910-shLSD1 cells were treated or not treated with Dox (100 ng/ml) for $48 \mathrm{~h}$ and LSD1 mRNA levels were detected via qRT-PCR. ${ }^{* *} \mathrm{P}<0.01{ }^{* * * *} \mathrm{P}<0.001$. NS, not significant. (B) HO8910-shLSD1 cells were treated or not treated with Dox $(100 \mathrm{ng} / \mathrm{ml})$ for $48 \mathrm{~h}$, after which the levels of LSD1 protein and H3K4 methylation were analyzed by western blotting. $\alpha$-tubulin and histone H3, loading control. (C) The shLSD1 cells were incubated with $100 \mathrm{ng} / \mathrm{ml}$ Dox for the indicated time-points or (D) different doses of Dox for $48 \mathrm{~h}$, and then the indicated antibodies were monitored by western blotting. Histone $\mathrm{H} 3$ was used as a loading control for $\mathrm{H} 3 \mathrm{~K} 4 \mathrm{me} 2$ and $\alpha$-tubulin was used as a loading control for other proteins. ${ }^{*} \mathrm{P}<0.05$ vs. untreated group. (E) The shLSD1 cells were incubated with different doses of Dox for $48 \mathrm{~h}$, after which LC3 and LSD1 mRNA levels were detected via qRT-PCR. "P<0.05 vs. Dox $0 \mathrm{ng} / \mathrm{ml}$ group. (F) HO8910 cells were treated with $100 \mu \mathrm{M}$ TCP for $24 \mathrm{~h}$, after which the levels of indicated proteins and H3K4 methylation were analyzed by western blotting. $\alpha$-tubulin and histone H3, loading control. (G) HO8910 cells were treated with $100 \mu \mathrm{M} \mathrm{TCP}$ for $24 \mathrm{~h}$, and the LC3 mRNA levels were detected via qRT-PCR. NS, not significant. Data shown are representative of three independent experiments. 
A
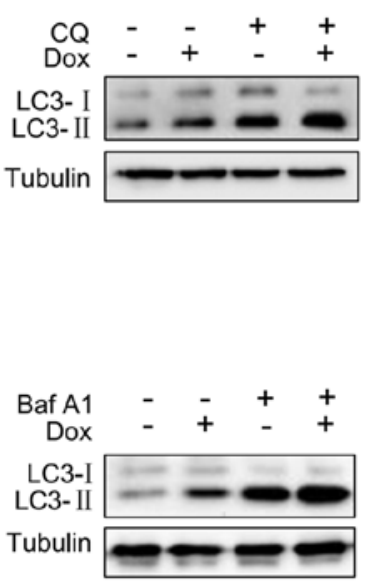

C

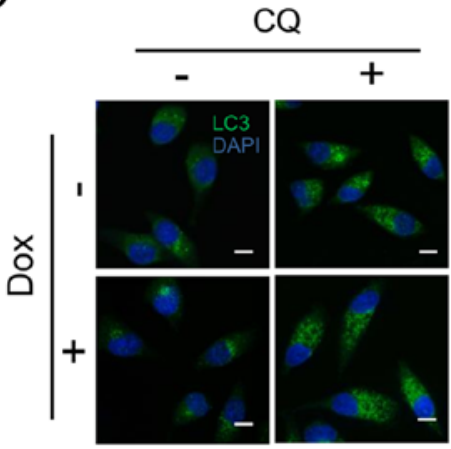

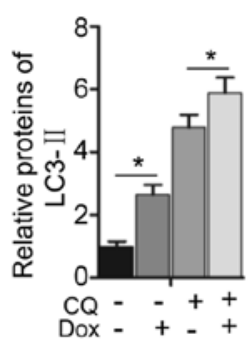

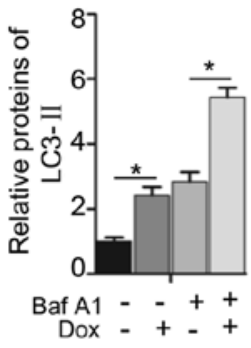

B
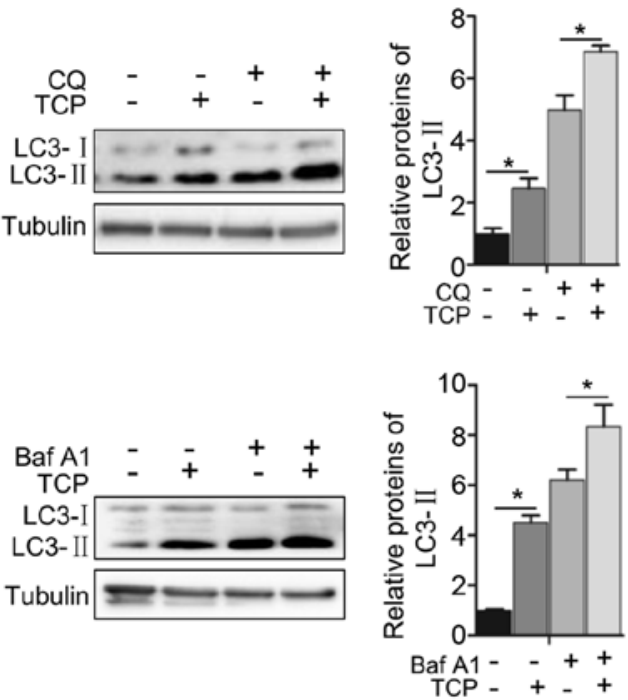

D

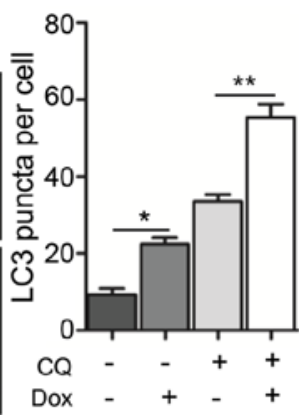

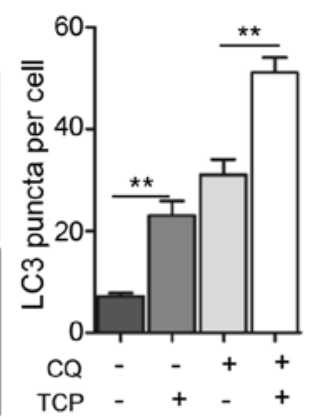

E
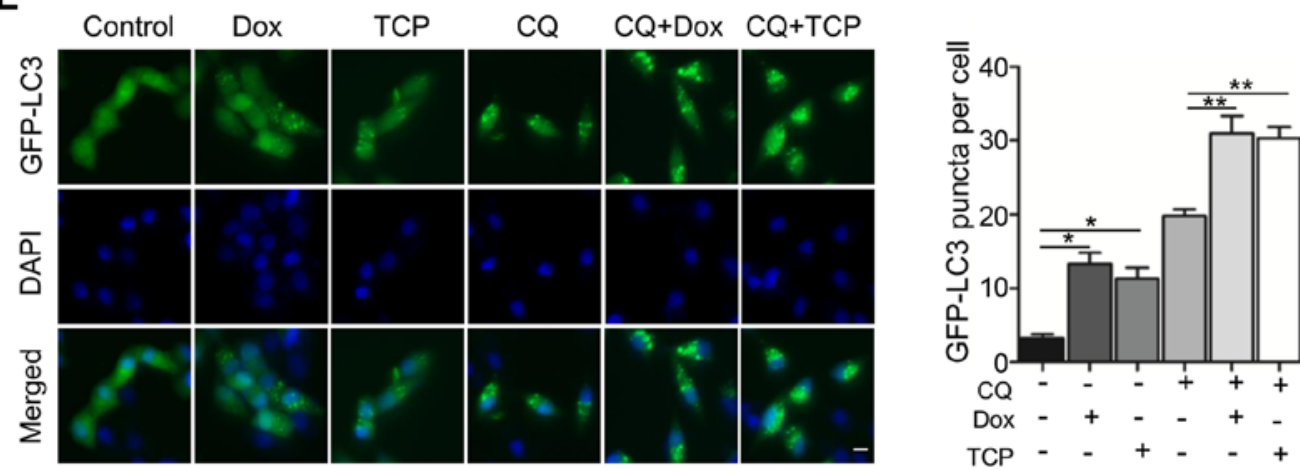

Figure 2. LSD1 depletion results in the induction of autophagic flux. (A and B) The Dox (A) or TCP (B) treated HO8910 cells were pretreated for $3 \mathrm{~h}$ with autophagy inhibitors $0.5 \mu \mathrm{M}$ BafA1 or $50 \mu \mathrm{M} \mathrm{CQ}$ and the proteins of interest were detected by western blotting. $\alpha$-tubulin, loading control. ${ }^{*} \mathrm{P}<0.05$. (C and $\left.\mathrm{D}\right) \mathrm{The}$ HO8910-shLSD1 (C) or HO8910 (D) cells were analyzed by immunofluorescence for LC3 (green) after treatment with $100 \mathrm{ng} / \mathrm{ml}$ Dox (C) or $100 \mu \mathrm{M} \mathrm{TCP}$ (D) along with pretreated for $3 \mathrm{~h}$ with $50 \mu \mathrm{M} \mathrm{CQ}$. The nucleus was stained by DAPI (blue). Scale bars, $50 \mu \mathrm{m}$. ${ }^{*} \mathrm{P}<0.05,{ }^{* * *} \mathrm{P}<0.01$. (E) Lentiviral vector-loaded GFP-LC3 was stably transfected in the HO8910 or HO8910-shLSD1 cells. The same treatment was followed as in the experiment above-mentioned in C and D. The GFP-LC3-positive autophagosomes were visualized under an inverted fluorescence microscope. Scale bars, $10 \mu \mathrm{m} .{ }^{*} \mathrm{P}<0.05$, ${ }^{* *} \mathrm{P}<0.01$. The numbers of the LC3 or GFP-LC3 fluorescent puncta were counted and assessed with ImageJ program (National Institutes of Health, Bethesda, MD, USA) for statistical analysis The statistical analysis was performed based on the numbers of the puncta in each of the cells. Data shown are representative of three independent experiments.

(Fig. 1B). Depletion of LSD1 caused significant accumulation of LC3-II in a time- and dose-dependent manner, but did not affect the levels of Beclin1 (Fig. 1C and D), a protein involved in autophagosomal biogenesis (26). In addition, knockdown of LSD1 did not significantly alter the LC3 mRNA levels (Fig. 1E), indicating that the effect of LSD1 downregulation on the LC3-II level was produced by affecting the protein translation, modification or degradation. To further confirm this result, we used tranylcypromine (TCP), a specific inhibitor of LSD1, to suppress the demethylase activity of LSD1 (27). TCP markedly increased the H3K4me2 levels, indicating that the demethylase activity of LSD1 was effectively inhibited 
A

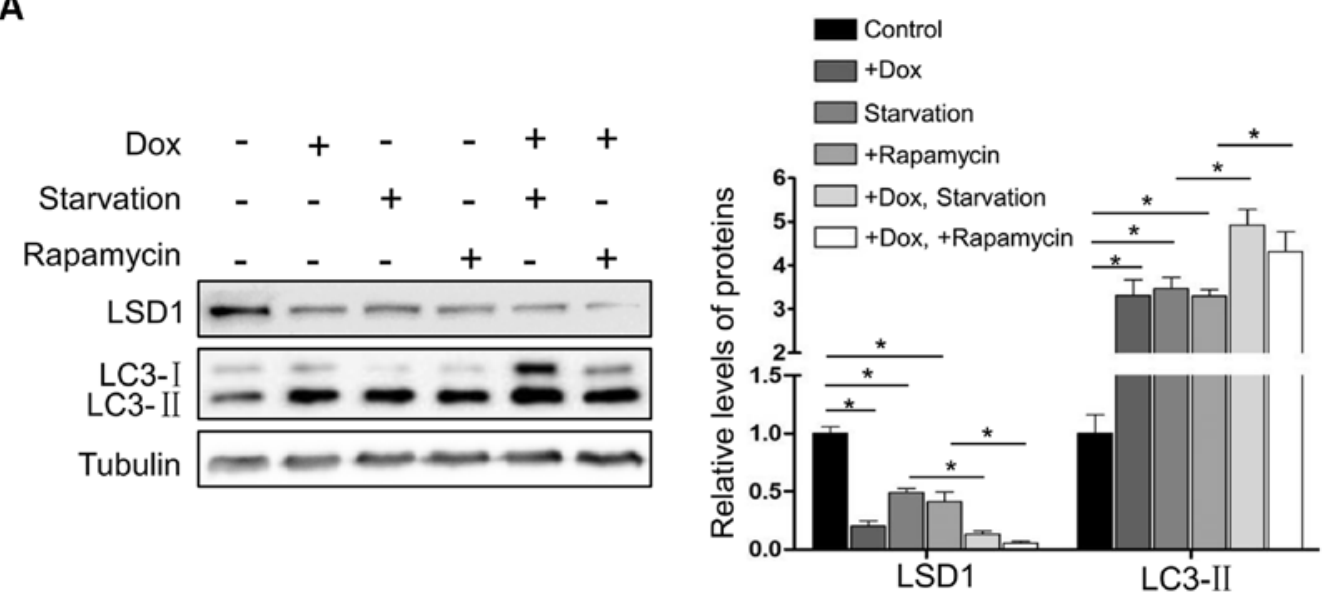

B
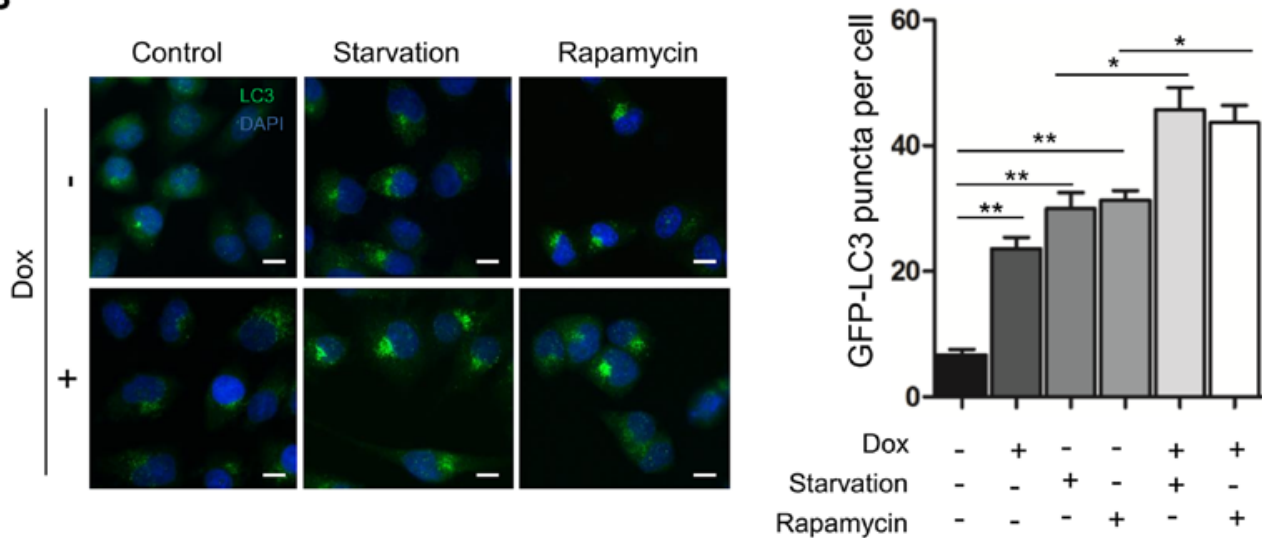

Figure 3. Inhibition of LSD1 enhances the activation of autophagy. (A) HO8910-shLSD1 cells were incubated in medium without serum for 16 h or 50 nM rapamycin for $12 \mathrm{~h}$ followed by treatment with or without $100 \mathrm{ng} / \mathrm{ml}$ Dox for $48 \mathrm{~h}$, and then the expression of LSD1 and LC3-II was determined by western blotting. $\alpha$-tubulin, loading control. ${ }^{*} \mathrm{P}<0.05$. (B) Immunofluorescence analysis of the experiments in A. The nucleus was stained by DAPI (blue). Scale bars, $50 \mu \mathrm{m}$. The numbers of the LC3 fluorescent puncta were counted and assessed with ImageJ program for statistical analysis. The statistical analysis was performed based on the numbers of the puncta in each of the cells. ${ }^{*} \mathrm{P}<0.05,{ }^{* *} \mathrm{P}<0.01$. Data shown are representative of three independent experiments.

(Fig. 1F). Consistent with the effect of the knockdown of LSD1, inhibition of LSD1 by TCP markedly increased the LC3-II protein levels (Fig. 1F), while it did not alter the mRNA levels (Fig. 1G). These data indicated that LSD1 was a negative regulator for production of LC3-II, indicating that LSD1 may play an important role in autophagy.

LSD1 depletion results in the induction of autophagic flux. To confirm the role of LSD1 in autophagy, we examined the effect of the downregulation or inhibition of LSD1 on autophagic flux. We used the V-ATPase inhibitor bafilomycin A1 (BafA1) and the lysosomal inhibitor chloroquine (CQ) to block the autophagic flux. As displayed in Fig. 2, BafA1 or CQ alone with the expression of LSD1 shRNA produced a significant increase of LC3-II (Fig. 2A). Similarly, BafA1 or CQ with the LSD1 inhibitor TCP significantly induced higher levels of LC3-II (Fig. 2B), indicating that the downregulation of the LSD1 activity or protein level stimulated autophagy. Subsequently, we determined the effect on autophagy flux by immunofluorescence staining of LC3. As displayed in Fig. 2C, knockdown of LSD1 increased the amount of LC3 puncta, which was consistent with the immunoblotting data displayed in Fig. 2A, indicating an inhibitory role of LSD1 in autophagy.
Treatment with CQ alone resulted in the accumulation of LC3 puncta, indicating that CQ blocks the autophagic flux. Knockdown of LSD1 plus treatment with CQ caused a greater increase in the amount of LC3 puncta than that of LSD1 knckdown or CQ treatment alone (Fig. 2C), indicating that depletion of LSD1 activated the autophagic flux. Treatment of the cells with the LSD1 inhibitor TCP revealed the same effect as that of LSD1 knockdown (Fig. 2D), indicating that the demethylase activity of LSD1 was required for the inhibitory effect on autophagy flux. We further confirmed the inhibitory role of LSD1 in autophagy flux by visualizing the effect of LSD1 knockdown or treatment with TCP on the amount of GFP-LC3 puncta in HO8910-shLSD1 cell line, as displayed in Fig. 2E. Collectively, these results clearly indicated that LSD1 was a negative regulator in autophagy.

Inhibition of LSDI enhances the activation of autophagy. Subsequently, we determined the effect of LSD1 on the activation of autophagy. Serum starvation and rapamycin are two known physiologic stimuli for the activation of autophagy $(12,28)$. As expected, serum withdrawal and rapamycin treatment induced the elevation of LC3-II (Fig. 3A) and increased the number of LC3 puncta (Fig. 3B) in HO8910 cells, indicating 
A

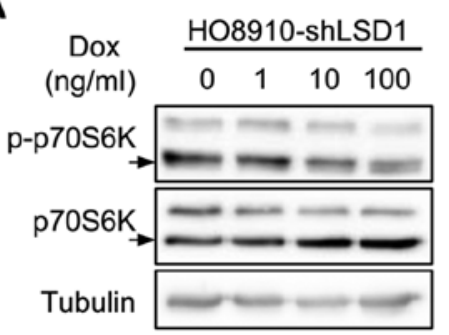

B

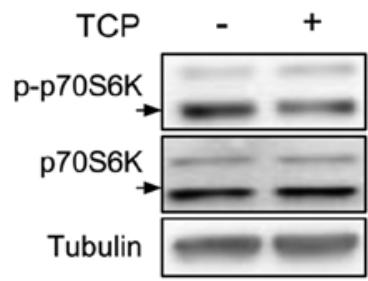

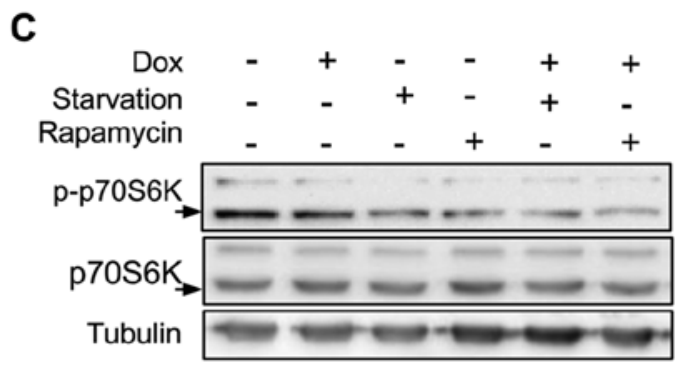

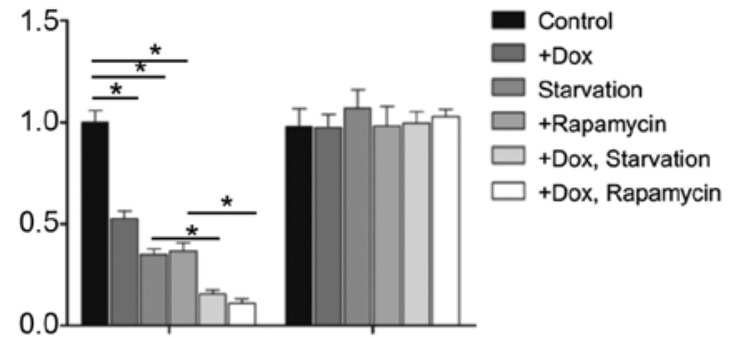

D

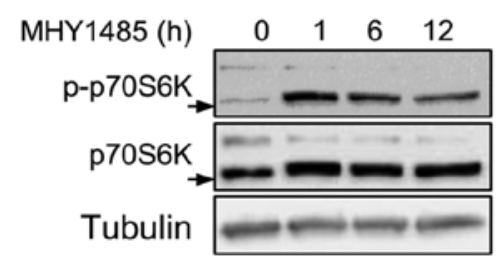

E
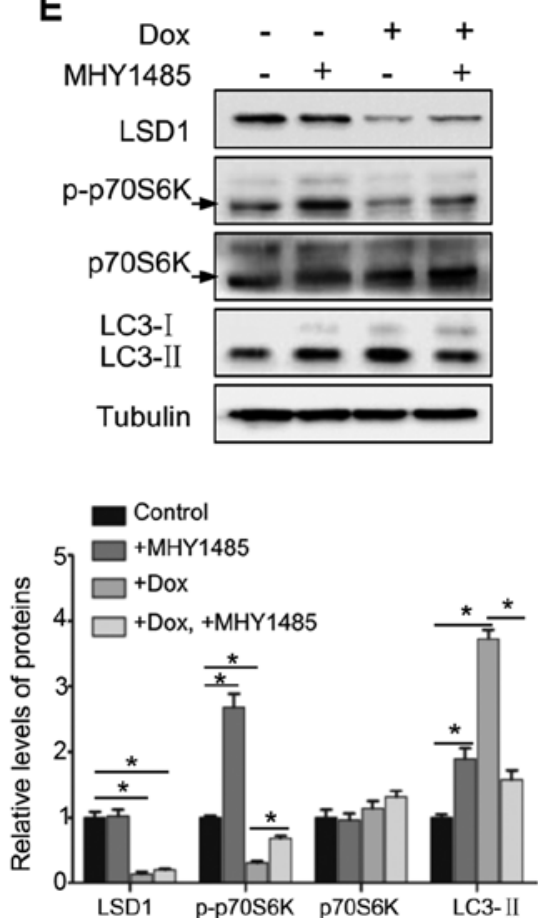

$\mathbf{F}$
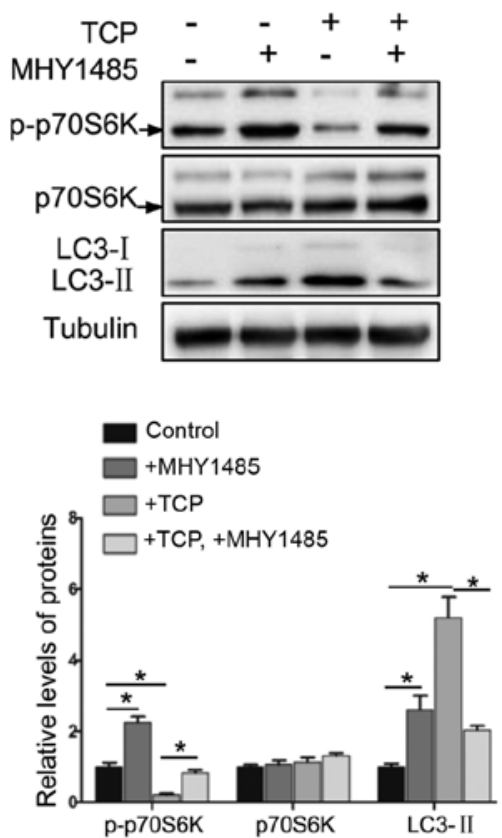

Figure 4. LSD1 regulates autophagy through the mTOR signaling pathway. (A) The HO8910-shLSD1 cells were treated with different doses of Dox for $48 \mathrm{~h}$. Phosphorylated p70S6K (p-p70S6K; Thr389) and total p70S6K were probed by western blotting. (B) HO8910 cells were treated with $100 \mu \mathrm{M}$ TCP for $24 \mathrm{~h}$, and phosphorylated p70S6K (p-p70S6K; Thr389) and total p70S6K were probed by western blotting. (C) HO8910-shLSD1 cells were incubated in medium without serum for $16 \mathrm{~h}$ or $50 \mathrm{nM}$ rapamycin for $12 \mathrm{~h}$ followed by treatment with or without $100 \mathrm{ng} / \mathrm{ml}$ Dox for $48 \mathrm{~h}$ and the proteins of interest were detected by western blotting. ${ }^{*} \mathrm{P}<0.05$. (D) The HO8910-shLSD1 cells were treated with $10 \mu \mathrm{M}$ mTOR specific activator MHY1485 for the indicated time-points. The expression of p-p70S6K and p70S6K was detected via western blot analysis. (E) The HO8910-shLSD1 cells were pretreated with $10 \mu \mathrm{M}$ MHY1485 for $1 \mathrm{~h}$ prior to the addition of Dox and the proteins of interest were detected via western blotting. ${ }^{*} \mathrm{P}<0.05$. (F) The HO8910 cells were pretreated with $10 \mu \mathrm{M}$ MHY1485 for $1 \mathrm{~h}$ prior to the addition of TCP and the proteins of interest were detected via western blotting. ${ }^{*} \mathrm{P}<0.05$. $\alpha$-tubulin, loading control. Data shown are representative of three independent experiments.

that serum starvation and rapamycin activated autophagy. Knockdown of LSD1 in cells further enhanced serum starvation- or rapamycin-induced elevation of LC3-II (Fig. 3A) and increased the number of LC3 puncta in shLSD1 cells (Fig. 3B), indicating that depletion of LSD1 promoted the starvation- and rapamycin-induced activation of autophagy. Collectively, these results demonstrated that LSD1 was a suppressor for autophagy activation.

Notably, serum starvation or rapamycin treatment caused a significant downregulation of LSD1 to the same level as that 
of shLSD1 (lanes 3 and 4; top panel; Fig. 3A), and additional downregulation of LSD1 was observed when the treatment was combined with the induction of the shLSD1 knockdown (lanes 5 and 6; the top panel; Fig. 3A), raising a possibility that serum starvation or rapamycin activated autophagy through the downregulation of LSD1, or LSD1 mediated the inactivation of autophagy by mTOR signaling.

LSD1 regulates autophagy through the mTOR signaling pathway. To explore the mechanism underlying the inhibitory effect of LSD1 on autophagy, we determined whether LSD1 was involved in the mTOR signaling pathway, a known pathway inactivating autophagy (16). As p70S6K is a known downstream substrate of mTOR (29), we detected phosphorylation of Thr389 in p70S6K to evaluate the mTOR activation. As displayed in Fig. 4A, phosphorylation of p70S6K (Thr389), was decreased in LSD1-depleted cells in a dose-dependent manner. Similarly, inhibition of mTOR signaling was also detected with treatment of the LSD1 inhibitor TCP (Fig. 4B). To further confirm these results, we determined the phosphorylation of p70S6K (Thr389) following starvation or rapamycin treatment in HO8920-shLSD1 cells and observed that knockdown of LSD1 resulted in further suppression of the mTOR pathway during starvation or rapamycin treatment (Fig. 4C), indicating that LSD1 regulated autophagy was indispensable through the mTOR signaling. To determine whether LSD1 knockdown induced autophagy directly via the mTOR pathway, we used an mTOR activator MHY1485 (30), which directly bound to mTOR and induced the phosphorylation of p70S6K (Thr389) to activate mTOR. As displayed in Fig. 4D, treatment with MHY1485 reached the maximum to activate mTOR at $1 \mathrm{~h}$ and was sustained for $12 \mathrm{~h}$. Notably, MHY1485 treatment abolished the effect of LSD1 knockdown on p70S6K phosphorylation and LC3-II accumulation (Fig. 4E), indicating that LSD1 knockdown-induced autophagy was blocked by mTOR activation. Similar results were also observed with TCP treatment (Fig. 4F). These results indicated that downregulation of LSD1 induced autophagy via the inhibition of mTOR signaling.

\section{Discussion}

In the present study, we revealed that LSD1, a lysine-specific demethylase, was a negative regulator of autophagy protein in HO8910 ovarian cancer cells. LSD1 reduction led to the activation of autophagy and the promotion of starvationand rapamycin-induced autophagy via the mTOR signaling pathway, which could be a therapeutic target for treatment of ovarian cancer due to the negative role of LSD1 in autophagy.

We have previously identified that LSD1 was overexpressed in ovarian cancer cells and its overexpression correlated with high migration and invasion (9,31). Furthermore, it has been reported that the autophagy level was lower in ovarian cancers than benign tumors and the induction of autophagy in this type of cancer was associated with prolonged overall survival of patients $(32,33)$. Thus, determining the regulatory mechanism of LSD1-mediated autophagy in ovarian cancer is urgently required for developing new therapeutic approaches. In fact, recent research has pointed the vital role of LSD1 in autophagy. For example, the FGF19-SHP-LSD1 regulatory axis mediated a postprandial epigenetic suppression of hepatic autophagy (34). Furthermore, a recent study reported that LSD1 overexpression promoted tumorigenesis and inhibited autophagy via decreasing the p62 protein stability in a demethylation-independent manner in gynecologic malignancies. In addition, the higher p62 and autophagic flux levels elicited by LSD1 inhibition could reduce tumor growth in vivo (21), indicating that LSD1 was an important regulator of autophagy in tumors, and further potential mechanisms of these effects need to be elucidated. In the present study, we demonstrated that LSD1 knockdown induced the accumulation of LC3-II proteins in HO8910 ovarian cancer cells. To confirm whether the LSD1 knockdown stimulated the production of autophagosomes or caused a blockage of autophagic degradation, we used two lysosomal inhibitors BafA1, that prevented maturation of autophagic vacuoles by inhibiting fusion between autophagosomes and lysosomes (35) and CQ, that raised the lysosomal $\mathrm{pH}$, which also inhibited fusion of autophagosomes with lysosomes (36). Upon treatment with these lysosomal inhibitors, an increased accumulation of LC3-II and autophagic flux were observed during LSD1 depletion, indicating that the LC3-II accumulation mediated by LSD1 knockdown was involved in the autophagy induction process.

The experiments presented in our study added a new mechanism underlying the LSD1-mediated suppression of autophagy. We revealed for the first time, that knockdown or inhibition of LSD1 induced autophagic flux in HO8910 ovarian cancer cells. While autophagy can be potently activated by serum starvation and rapamycin, we demonstrated that depletion of LSD1 obviously promoted the starvation- and rapamycin-induced activation of autophagy, which clearly demonstrated that LSD1 was a suppressor for autophagy activation. Notably, we observed that serum starvation or rapamycin treatment downregulated LSD1, indicating that downregulation of LSD1 may mediate the activation of autophagy or may have a feedback effect on LSD1 expression in starvation- and rapamycin-induced autophagy.

The mTOR signaling pathway plays a crucial role in regulating autophagy, and mTOR is inhibited in response to various cellular conditions such as serum starvation, stress stimulation and growth factor deprivation (16). Several studies have indicated that the mTOR signaling pathway may be involved in epigenetic changes during autophagy. For example, methyltransferase EZH2 epigenetically suppressed several negative regulators of mTOR pathway via MTA2 to inhibit autophagy (37). Furthermore, LSD1 has been found to be a regulator of the mTOR pathway in neuroblastoma cells. Ambrosio et al (39) found that SESN2, which serves as a key positive regulator of the autophagic pathway by directly inhibiting the mTORC1 activity (38), played an important role in LSD1-mediated autophagy. SESN2 is an LSD1-suppressed target gene and LSD1 inhibition triggered the transcriptional activation of SESN2, which resulted in the decrease of mTORC1 activity (39). Collectively, these findings provided a rationale for our hypothesis and indicated that the molecular mechanism of LSD1-mediated autophagy in ovarian cancer should be urgently explored. In the present study, we demonstrated that LSD1 depletion-induced autophagy in ovarian cancer cells was dependent on mTOR inactivation which was characterized by decreasing the phosphorylation of p70S6K (Thr389), a downstream effector and marker of mTOR. 
Furthermore, we noticed that the mTOR activator MHY1485 rescued the phosphorylation levels of p70S6K in response to LSD1 deficiency, which further supported our hypothesis. In addition, LSD1 depletion-induced LC3-II accumulation was attenuated upon mTOR activation. Thus, LSD1 appeared to regulate autophagy in an mTOR-dependent way.

In conclusion, our data identified LSD1 as an autophagy suppressor in HO8910 cells, which involved both basal and starvation- or rapamycin- induced autophagic progress through the mTOR pathway. Further studies are warranted to elucidate the potential mechanisms of how starvation and rapamycin alter the expression of LSD1, as well as uncover the LSD1-dependent epigenetic changes which may mediate autophagic process in ovarian cancer. Our results established a novel link among epigenetic regulation, mTOR pathway and autophagy in HO8910 ovarian cancer cells, providing new insights for future cancer treatment.

\section{Acknowledgements}

Not applicable.

\section{Funding}

The present study was supported by a grant from the National Natural Science Foundation of China (no. 81170573).

\section{Availability of data and materials}

The datasets used during the present study are available from the corresponding author upon reasonable request.

\section{Authors' contributions}

GS and QL conceived and designed the study. YW performed the experiments. YW and TH wrote the paper. TH, RW, JW and KP were involved in the conception of the study. All authors read and approved the manuscript and agree to be accountable for all aspects of the research in ensuring that the accuracy or integrity of any part of the work are appropriately investigated and resolved.

\section{Ethics approval and consent to participate}

Not applicable.

\section{Consent for publication}

Not applicable.

\section{Competing interests}

The authors state that they have no competing interests.

\section{References}

1. Lawrence M, Daujat S and Schneider R: Lateral Thinking: How histone modifications regulate gene expression. Trends Genet 32: 42-56, 2016.

2. Baek SH: When signaling kinases meet histones and histone modifiers in the nucleus. Mol Cell 42: 274-284, 2011.
3. Shi Y and Whetstine JR: Dynamic regulation of histone lysine methylation by demethylases. Mol Cell 25: 1-14, 2007.

4. Lan F, Nottke AC and Shi Y: Mechanisms involved in the regulation of histone lysine demethylases. Curr Opin Cell Biol 20: 316-325, 2008.

5. Serce N, Gnatzy A, Steiner S, Lorenzen H, Kirfel J and Buettner R: Elevated expression of LSD1 (Lysine-specific demethylase 1) during tumour progression from pre-invasive to invasive ductal carcinoma of the breast. BMC Clin Pathol 12: 13, 2012.

6. Hayami S, Kelly JD, Cho HS, Yoshimatsu M, Unoki M, Tsunoda T, Field HI, Neal DE, Yamaue H, Ponder BA, et al: Overexpression of LSD1 contributes to human carcinogenesis through chromatin regulation in various cancers. Int J Cancer 128: 574-586, 2011.

7. Guo WH, Yuan LH, Xiao ZH, Liu D and Zhang JX: Overexpression of SUMO-1 in hepatocellular carcinoma: A latent target for diagnosis and therapy of hepatoma. J Cancer Res Clin Oncol 137: 533-541, 2011.

8. Shao G, Wang J, Li Y, Liu X, Xie X, Wan X, Yan M, Jin J, Lin Q, Zhu H, et al: Lysine-specific demethylase 1 mediates epidermal growth factor signaling to promote cell migration in ovarian cancer cells. Sci Rep 5: 15344, 2015.

9. Li Y, Wan X, Wei Y, Liu X, Lai W, Zhang L, Jin J, Wu C, Shao Q, Shao G, et al: LSD1-mediated epigenetic modification contributes to ovarian cancer cell migration and invasion. Oncol Rep 35: 3586-3592, 2016.

10. Klionsky DJ: Autophagy: From phenomenology to molecular understanding in less than a decade. Nat Rev Mol Cell Biol 8: 931-937, 2007.

11. Mizushima N: Autophagy in protein and organelle turnover. Cold Spring Harb Symp Quant Biol 76: 397-402, 2011.

12. Johansen $T$ and Lamark T: Selective autophagy mediated by autophagic adapter proteins. Autophagy 7: 279-296, 2011.

13. Mizushima N, Levine B, Cuervo AM and Klionsky DJ: Autophagy fights disease through cellular self-digestion. Nature 451: 1069-1075, 2008.

14. Kabeya Y, Mizushima N, Ueno T, Yamamoto A, Kirisako T, Noda T, Kominami E, Ohsumi Y and Yoshimori T: LC3, a mammalian homologue of yeast Apg8p, is localized in autophagosome membranes after processing. EMBO J 19: 5720-5728, 2000 .

15. Tanida I, Ueno T and Kominami E: LC3 conjugation system in mammalian autophagy. Int J Biochem Cell Biol 36: 2503-2518, 2004.

16. Ravikumar B, Vacher C, Berger Z, Davies JE, Luo S, Oroz LG, Scaravilli F, Easton DF, Duden R, O'Kane CJ, et al: Inhibition of mTOR induces autophagy and reduces toxicity of polyglutamine expansions in fly and mouse models of Huntington disease. Nat Genet 36: 585-595, 2004.

17. Pimkina $J$ and Murphy ME: ARF, autophagy and tumor suppression. Autophagy 5: 397-399, 2009.

18. Janji B, Viry E, Baginska J, Moer KV and Berchem G: Role of autophagy in cancer and tumor progression. autophagy - A double-edged sword - Cell Survival or Death?: 189-190, 2013.

19. Polager S, Ofir M and Ginsberg D: E2F1 regulates autophagy and the transcription of autophagy genes. Oncogene 27: 4860-4864, 2008.

20. Settembre C, Di Malta C, Polito VA, Garcia Arencibia M, Vetrini F, Erdin S, Erdin SU, Huynh T, Medina D, Colella P, et al: TFEB links autophagy to lysosomal biogenesis. Science 332: 1429-1433, 2011.

21. Chao A, Lin CY, Chao AN, Tsai CL, Chen MY, Lee LY, Chang TC, Wang TH, Lai CH and Wang HS: Lysine-specific demethylase 1 (LSD1) destabilizes p62 and inhibits autophagy in gynecologic malignancies. Oncotarget 8: 74434-74450, 2017.

22. Byun S, Kim YC, Zhang Y, Kong B, Guo G, Sadoshima J, Ma J, Kemper B and Kemper JK: A postprandial FGF19-SHP-LSD1 regulatory axis mediates epigenetic repression of hepatic autophagy. EMBO J 36: 1755-1769, 2017.

23. Shao GB, Wang J, Zhang LP, Wu CY, Jin J, Sang JR, Lu HY, Gong AH, Du FY and Peng WX: Aging alters histone H3 lysine 4 methylation in mouse germinal vesicle stage oocytes. Reprod Fertil Dev 27: 419-426, 2015.

24. Livak KJ and Schmittgen TD: Analysis of relative gene expression data using real-time quantitative PCR and the $2(-\Delta \Delta \mathrm{C}(\mathrm{T}))$ Method. Methods 25: 402-408, 2001.

25. Ren S, Ouyang DY, Saltis M, Xu LH, Zha QB, Cai JY and He XH: Anti-proliferative effect of 23,24-dihydrocucurbitacin F on human prostate cancer cells through induction of actin aggregation and cofilin-actin rod formation. Cancer Chemother Pharmacol 70: 415-424, 2012. 
26. Liang XH, Jackson S, Seaman M, Brown K, Kempkes B, Hibshoosh $\mathrm{H}$ and Levine B: Induction of autophagy and inhibition of tumorigenesis by beclin 1. Nature 402: 672-676, 1999.

27. Schenk T, Chen WC, Göllner S, Howell L, Jin L, Hebestreit K, Klein HU, Popescu AC, Burnett A, Mills K, et al: Inhibition of the LSD1 (KDM1A) demethylase reactivates the all-transretinoic acid differentiation pathway in acute myeloid leukemia. Nat Med 18: 605-611,2012.

28. Kobayashi S, Kishimoto T, Kamata S, Otsuka M, Miyazaki M and Ishikura $\mathrm{H}$ : Rapamycin, a specific inhibitor of the mammalian target of rapamycin, suppresses lymphangiogenesis and lymphatic metastasis. Cancer Sci 98: 726-733, 2007.

29. Ding J, Zhang ZM, Xia Y, Liao GQ, Pan Y, Liu S, Zhang Y and Yan ZS: LSD1-mediated epigenetic modification contributes to proliferation and metastasis of colon cancer. Br J Cancer 109: 994-1003, 2013.

30. Choi YJ, Park YJ, Park JY, Jeong HO, Kim DH, Ha YM, Kim JM, Song YM, Heo HS, Yu BP, et al: Inhibitory effect of mTOR activator MHY1485 on autophagy: Suppression of lysosomal fusion. PLoS One 7: e43418, 2012.

31. Lv T, Yuan D, Miao X, Lv Y, Zhan P, Shen X and Song Y: Over-expression of LSD1 promotes proliferation, migration and invasion in non-small cell lung cancer. PLoS One 7: e35065, 2012.

32. Shen Y, Li DD, Wang LL, Deng R and Zhu XF: Decreased expression of autophagy-related proteins in malignant epithelial ovarian cancer. Autophagy 4: 1067-1068, 2008.
33. Bartholomeusz C, Rosen D, Wei C, Kazansky A, Yamasaki F, Takahashi T, Itamochi H, Kondo S, Liu J and Ueno NT: PEA-15 induces autophagy in human ovarian cancer cells and is associated with prolonged overall survival. Cancer Res 68: 9302-9310, 2008

34. Byun S, Kim YC, Zhang Y, Kong B, Guo G, Sadoshima J, Ma J, Kemper B and Kemper JK: A postprandial FGF19 - SHP LSD1 regulatory axis mediates epigenetic repression of hepatic autophagy. EMBO J 36: 1755-1769, 2017.

35. Yamamoto A, Tagawa Y, Yoshimori T, Moriyama Y, Masaki R and Tashiro Y: Bafilomycin A1 prevents maturation of autophagic vacuoles by inhibiting fusion between autophagosomes and lysosomes in rat hepatoma cell line, H-4-II-E cells. Cell Struct Funct 23: 33-42, 1998.

36. Mizushima $\mathrm{N}$ and Yoshimori T: How to interpret LC3 immunoblotting. Autophagy 3: 542-545, 2007.

37. Füllgrabe J, Klionsky DJ and Joseph B: The return of the nucleus: Transcriptional and epigenetic control of autophagy. Nat Rev Mol Cell Biol 15: 65-74, 2014.

38. Wolfson RL, Chantranupong L, Saxton RA, Shen K, Scaria SM, Cantor JR and Sabatini DM: Sestrin2 is a leucine sensor for the mTORC1 pathway. Science 351: 43-48, 2016.

39. Ambrosio S, Saccà CD, Amente $\mathrm{S}$, Paladino $\mathrm{S}$, Lania L and Majello B: Lysine-specific demethylase LSD1 regulates autophagy in neuroblastoma through SESN2-dependent pathway. Oncogene 36: 6701-6711, 2017. 